TITLE:

\title{
Shear-induced phase separation in ""nonentangled"" oligomer mixture
}

$\operatorname{AUTHOR}(S)$ :

Takenaka, M; Miyazawa, M; Nishitsuji, S; Hashimoto, $T$

\section{CITATION:}

Takenaka, M ...[et al]. Shear-induced phase separation in

""nonentangled"" oligomer mixture. JOURNAL OF CHEMICAL PHYSICS 2004, 121(15): 7501-7504

ISSUE DATE:

2004-10-15

URL:

http://hdl.handle.net/2433/39760

\section{RIGHT:}

Copyright 2004 American Institute of Physics. This article may be downloaded for personal use only. Any other use requires prior permission of the author and the American Institute of Physics. 


\title{
Comparison in fractal dimension between those obtained from structure factor and viscoelasticity of gel networks of 1,3:2,4-bis- $O$-( $p$-methylbenzylidene)- $D$-sorbitol in polystyrene melt at gel point
}

\author{
Mikihito Takenaka \\ Department of Polymer Chemistry, Graduate School of Engineering, Kyoto University, Nishikyo-ku, \\ Kyoto 615-8510, Japan \\ Toshiaki Kobayashi \\ Research and Development Division, New Japan Chemical Co., 13 Yoshijima, Yagura-Cho, Fushimi-Ku, \\ Kyoto 612-8224, Japan \\ Kenji Saijo, Hirokazu Tanaka, Naoki Iwase, and Takeji Hashimotoa) \\ Department of Polymer Chemistry, Graduate School of Engineering, Kyoto University, Nishikyo-ku, \\ Kyoto 615-8510, Japan \\ Masaoki Takahashi \\ Department of Polymer Science and Engineering, Kyoto Institute of Technology, Matsugasaki, \\ Kyoto 606-8585, Japan
}

(Received 19 April 2004; accepted 17 May 2004)

\begin{abstract}
We investigated time evolution of shear moduli in the physical gelation process of 1,3:2,4-bis- $O$-( $p$-methylbenzylidene)- $D$-sorbitol in polystyrene melt. At the gel point, storage and loss shear moduli, $G^{\prime}$ and $G^{\prime \prime}$, were described by the power law of frequency $\omega, G^{\prime} \sim G^{\prime \prime} \sim \omega^{n}$, with the critical exponent $n$ being nearly equal to $2 / 3$, in agreement with the value predicted by the percolation theory. We also investigated the structure factor over two decades in length scale at gel point by using ultra-small-angle X-ray scattering, and small-angle X-ray scattering. We found the power-law behavior in low- $q$ region, indicating that the gel network forms the self-similar structure with mass-fractal dimension. Comparison between the exponent of mass-fractal dimension from structure factor and that from viscoelasticity indicates that hydrodynamic interactions are completely screened out and the excluded volume effects are dominant in the gel. The gel strength was found to increase with the decrease in the lower limit length scale of fractality. (C) 2004 American Institute of Physics. [DOI: 10.1063/1.1770590]
\end{abstract}

\section{INTRODUCTION}

1,3:2,4-bis- $O$-( $p$-methylbenzylidene)- $D$-sorbitol (PDTS) forms gels in organic solvents and/or polymer melts by less than 10 wt. \%. ${ }^{1}$ The gelation is expected to be due to the formation of the percolation network of PDTS molecules in the solvents or melts as a consequence of their intermolecular associations. The intermolecular association may further result in crystallization (solid-liquid phase transition) of PDTS in PDTS solutions. The sol-gel transition of PDTS solution is, thus, thermoreversible and the transition temperature decreases with increasing solubility of PDTS to the solvents or melts. In a previous paper, ${ }^{2}$ we have investigated the mechanism of sol-gel transition of PDTS polystyrene (PS) by means of rheological measurements in order to explore the mechanism of the gelation processes of PDTS/PS system as a typical physical gel. Similar to the chemical gel systems, we found that the frequency $\omega$ dependence of the storage modulus $G^{\prime}$ and the loss modulus $G^{\prime \prime}$ obeys the power law behavior $G^{\prime} \sim G^{\prime \prime} \sim \omega^{n}$ at the gel point. The obtained values

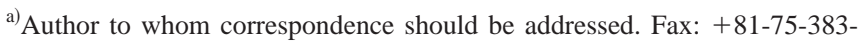
2623; Electronic mail: hashimoto@alloy.polym.kyoto-u.ac.jp
}

of $n$ are about 0.67 and independent of the gelation temperature $T$. They agree with the value $n=2 / 3$ predicted by the percolation theory without hydrodynamic interactions so that we concluded that the growth of the network structure of PDTS obeys the percolation theory which predicts that the fractal dimension $d_{f}$ of the network is 2.5. However, since this conclusion was obtained under the assumption that the hydrodynamic interactions can be neglected in the dynamics of the network, we need to obtain the fractal dimension by measuring the structure factor directly to confirm the validity of the percolation theory for the PDTS/PS system.

In order to investigate the fractal dimension, we need to measure the structure factor with wide range in length scale at the gel point. From the order of $1 \mathrm{~nm}$ to that of $100 \mathrm{~nm}$, we can use the time-resolved X-ray scattering and easily measure the structure factor at the gel point in situ. However, above the order of $100 \mathrm{~nm}$, we have to use Bonse-Hart type ultra-small-angle X-ray scattering so that the measurement of the structure factor is impossible with the wide range in length scale in situ. However, as for the PDTS/PS system, we can freeze the network structure of PDTS/PS system by quenching the sample below glass transition temperature of PS quickly so that we can precisely measure the structure 
factor of the frozen systems with wide range in length scale. Such a technique was successfully applied to the freeze of the structure of block copolymer in the disordered state. ${ }^{3}$ Thus, in this paper, we shall precisely measure the structure factor of PS/PDTS systems at the gel point by various scattering techniques. The objective of the present study is to compare the fractal dimension obtained by scattering function with that by the viscoelastic measurements to confirm the validity of the percolation theory in the growth of the network structure of PDTS in PS matrix.

We will describe the sample and experimental methods in Sec. II. In Secs. III A-III C, we will show the results of viscoelastic measurements and the fractal dimension. In Sec. III D, we will show the experimental results of the scattering measurements and compare the fractal dimensions. Finally, we will conclude our results in Sec. IV.

\section{EXPERIMENTAL SECTION}

\section{A. Samples}

The synthesis of PDTS was described in detail elsewhere. ${ }^{4}$ The weight-averaged molecular weight Mw and heterogeneity index $\mathrm{Mw} / \mathrm{Mn}$ of PS are, respectively, 6.4 $\times 10^{4}$ and 1.03 , where $\mathrm{Mn}$ is the number-average molecular weight. The concentration of PDTS in PDTS/PS is 2.5 wt. \%. PDTS/PS was first dissolved into a dioxane solution at $60{ }^{\circ} \mathrm{C}$ to prepare a homogeneous solution containing $10 \mathrm{wt} \%$ of PDTS/PS. The solution was then frozen at $-60^{\circ} \mathrm{C}$ for 40 min and dried for $40 \mathrm{~h}$ in vacuum in order to obtain the frozen homogeneous mixture of PDTS/PS free from dioxane. We molded the freeze-dried sample at $200^{\circ} \mathrm{C}$ and 150 $\mathrm{kg} / \mathrm{cm}^{2}$ into a disk with $2 \mathrm{~mm}$ thickness and $25 \mathrm{~mm}$ diameter, and the disk was then rapidly quenched into cold water. The as-prepared disk which is a vitrified homogeneous mixture of PDTS/PS was used for further experiments.

\section{B. Viscoelastic measurements}

Dynamic viscoelastic measurements have been made during gelation process using the Rheometrics Mechanical Spectrometer ${ }^{\circledR}$. We set the disk sample of PDTS/PS into parallel plates of $25 \mathrm{~mm}$ diameter and annealed at $220^{\circ} \mathrm{C}$ for 2 min to make the sample in sol state. Subsequently, we quenched the sample to 183,181 , and $178^{\circ} \mathrm{C}$, where the system undergoes the sol-gel transition. Note that the sol-gel transition temperature was determined to be $185^{\circ} \mathrm{C}$ in the cooling process. ${ }^{5}$ Then we measured frequency dependencies of storage and loss moduli, $G^{\prime}$ and $G^{\prime \prime}$, every $5 \mathrm{~min}$ at each frequency in the frequency range from 0.1 to $100 \mathrm{~s}^{-1}$. The strain was 0.1 , which is within the linear viscoelastic criterion. We also measured the frequency dependencies of $G^{\prime}$ and $G^{\prime \prime}$ for PS in the frequency range from 0.1 to $100 \mathrm{~s}^{-1}$ at 183,181 , and $178^{\circ} \mathrm{C}$.

\section{Scattering measurements}

In order to cover the wide length scale, we combined the data of small-angle X-ray scattering (SAXS), and ultrasmall-angle scattering (USAXS). The SAXS intensity with

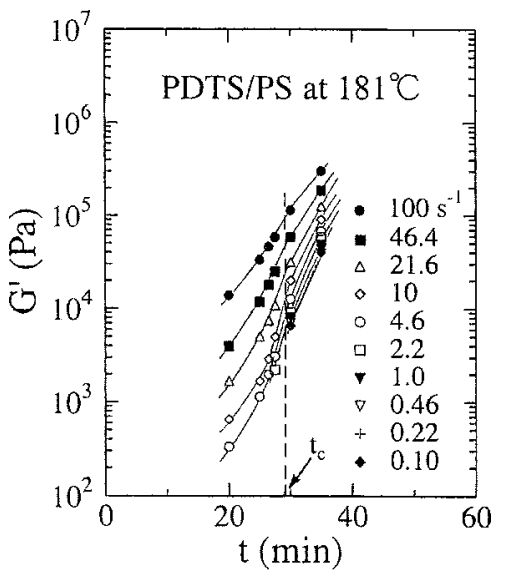

FIG. 1. Change in $G^{\prime}$ at fixed $\omega$ 's with $t$ during the gelation process of the PDTS/PS system at $181{ }^{\circ} \mathrm{C} . t_{c}$ is the gel point assessed from Fig. 3 .

the range of the magnitude of the scattering vector $q$ of $0.05<q<1.0 \mathrm{~nm}^{-1}$ was measured at BL45XU SAXS station, SPring- $8,{ }^{6}$ where $q$ is defined by

$$
q=(4 \pi / \lambda) \sin (\theta / 2)
$$

with $\lambda$ and $\theta$ being the wavelength and the scattering angle in the sample, respectively. The SAXS patterns were corrected for the background, air, and the transmittance and then circularly averaged for further analysis. The $q$ range of USAXS was $0.008<q<0.1 \mathrm{~nm}^{-1}$. The details of the apparatus have been described elsewhere. ${ }^{7}$ The USAXS profiles were corrected for the slit-height and slit-width smearing, the background, and the transmittance.

For SAXS and USAXS measurements, we molded the sample from the freeze dried one and then installed into the sample holders. In order to measure the scattering profiles at gel point, we made the measurements in the following way. As for the SAXS measurement, the time changes in the scattered intensity were observed with $10 \mathrm{sec}$ interval after the onset of the quench to 183,181 , and $178^{\circ} \mathrm{C}$. The measurement time is $1 \mathrm{sec} / \mathrm{scan}$. We used the scattering patterns at sol-gel points which were determined by the viscoelastic measurements. As for the USAXS measurement, we first annealed the samples at $200{ }^{\circ} \mathrm{C}$ for $20 \mathrm{~min}$ and then quenched the sample to 183,181 , and $178^{\circ} \mathrm{C}$ and annealed up to the sol-gel points. Subsequently, we quenched the samples below the glass transition temperature of PS by dunking the annealed samples into iced water to freeze the structure at the sol-gel points for the further USAXS measurement.

\section{RESULTS AND DISCUSSION}

\section{A. Change in viscoelasticity during gelation process with time}

Figures 1 and 2 show changes in $G^{\prime}$ and $G^{\prime \prime}$ with time at fixed $\omega$ 's after the onset of the gelation at $181^{\circ} \mathrm{C} . G^{\prime}$ and $G^{\prime \prime}$ increase with time at all observed $\omega$ regions, indicating that the PDTS aggregates and forms clusters of networks in PS matrix and that amount and size of the clusters of the networks formed increase with time. The increase in $G^{\prime}$ and $G^{\prime \prime}$ 


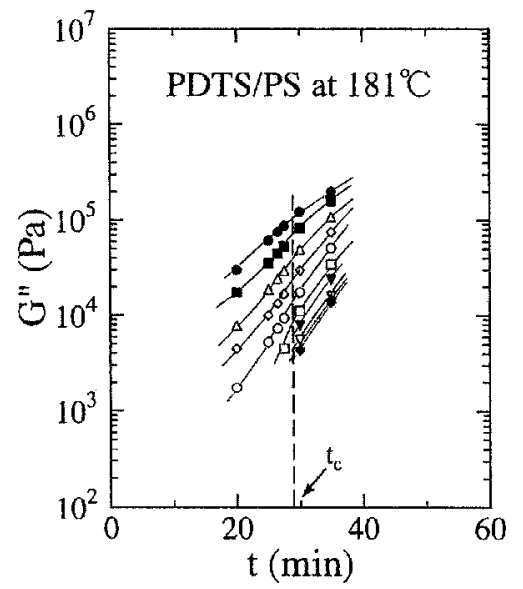

FIG. 2. Change in $G^{\prime \prime}$ at fixed $\omega^{\text {'s }}$ with $t$ during the gelation process of the PDTS/PS system at $181{ }^{\circ} \mathrm{C} . t_{c}$ is the gel point assessed from Fig. 3 .

is more rapid for smaller $\omega$, confirming the size growth of clusters with time. The vertical dashed line indicates the gel point $t_{c}$, as will be discussed later.

\section{B. Change in $\tan \delta$}

When networks grow, either via physical or chemical gelation process, with self-similarity before reaching the solgel transition point, the networks are characterized by an upper cut-off length of the clusters of the networks, $\xi$, and the fractal dimension $d_{f}$ (Refs. 8 and 9) of the networks. $\xi$ can be described by

$$
\xi \sim \epsilon^{-\nu},
$$

where $\epsilon$ and $\nu$ are, respectively, the relative distance of a point of observation in the sol state from the gel point and the critical exponent. The distance $\epsilon$ is given by

$$
\epsilon \equiv\left|p-p_{c}\right| / p_{c},
$$

where $p$ and $p_{c}$ are the extents of a "reaction" before the gel point and at the gel point, respectively. In our experiment, the extent of the reaction corresponds to the crystallinity of the PDTS induced by the solid-liquid transition. Before the gelation point, the zero-shear viscosity $\eta_{0}$, steady state compliance $J_{e}^{0}$, and the terminal relaxation time $\tau$ scale as

$$
\begin{aligned}
& \eta_{0} \sim \epsilon^{-k}, \\
& J_{e}^{0} \sim \epsilon^{-z},
\end{aligned}
$$

and

$$
\tau \sim \eta_{0} J_{e}^{0} \sim \epsilon^{-(k+z)} .
$$

In polymer systems where hydrodynamic interactions are completely screened out (corresponding to Rouse limit) and the excluded volume effects are dominant, the critical exponents $k$ and $z$ are given by ${ }^{8,9}$

$$
\begin{aligned}
& k=\nu\left(d_{f}+2-d\right), \\
& z=\nu d
\end{aligned}
$$

with $d_{f}$ and $d$ (=3 in our case) being the fractal dimension and space dimensionality, respectively. On the other hand, if excluded volume effects as well as hydrodynamic interactions are completely screened out, $k$ is expressed by

$$
k=\nu\left(\bar{d}_{f}+2-d\right)
$$

with

$$
\bar{d}_{f}=\frac{2 d_{f}}{d+2-2 d_{f}},
$$

while $z$ has the same form as Eq. (8).

At gel point $\left(p=p_{c}\right)$, stress relaxation moduli $G(t), G^{\prime}$, and $G^{\prime \prime}$ show the following power law behavior:

$$
G(t)=S t^{-n}
$$

and

$$
G^{\prime} \sim G^{\prime \prime} \sim \omega^{n},
$$

where gel strength $S$ characterizes the strength of the gel at gel point and is calculated from

$$
S=\frac{2 \Gamma(n) \sin (n \pi / 2) G^{\prime}(\omega)}{\pi \omega^{n}},
$$

where $\Gamma(n)$ is the Gamma function.

The power law is predicted by Martin, Adolf, and Wilcoxon ${ }^{8}$ based on the dynamic scaling theory and by Rubinstein, Colby, and Gillmor, ${ }^{9}$ Muthukumar, ${ }^{10,11}$ and Hess, Vilgis, and Winter ${ }^{12}$ by applying the Rouse dynamics. It postulates that the longest relaxation time of the gel diverges with the same critical exponent from both sides of the gel point; a general expression for $n$ is given by

$$
n=\frac{z}{k+z} \text {. }
$$

When hydrodynamic interactions are completely screened out and the excluded volume effects are dominant in the cluster, we obtain

$$
n=\frac{d}{d_{f}+2}
$$

from Eqs. (7), (8), and (14). On the other hand, if excluded volume effects as well as hydrodynamic interactions can be completely screened out, $n$ is expressed by ${ }^{11}$

$$
n=\frac{d\left(d+2-2 d_{f}\right)}{2\left(d+2-d_{f}\right)}
$$

from Eqs. (8)-(10) and (14). If the power law of Eq. (12) holds in sufficiently wide range of $\omega$ at gel point, the loss tangent, $\tan \delta\left[=G^{\prime \prime}(\omega) / G^{\prime}(\omega)\right]$, at gel point is given by

$$
\tan \delta=\tan \left(\frac{n \pi}{2}\right)
$$

from the Kramers-Kronig relation, ${ }^{13}$ indicating that $\tan \delta$ is independent of $\omega$ at gel point and its value depends only on $n$.

Figure 3 shows changes in $\tan \delta$ with time at fixed $\omega$ 's after the quench to $181^{\circ} \mathrm{C}$. We found the characteristic time $t_{c}=29.0 \mathrm{~min}$, where $\tan \delta$ becomes independent of $\omega$. From 


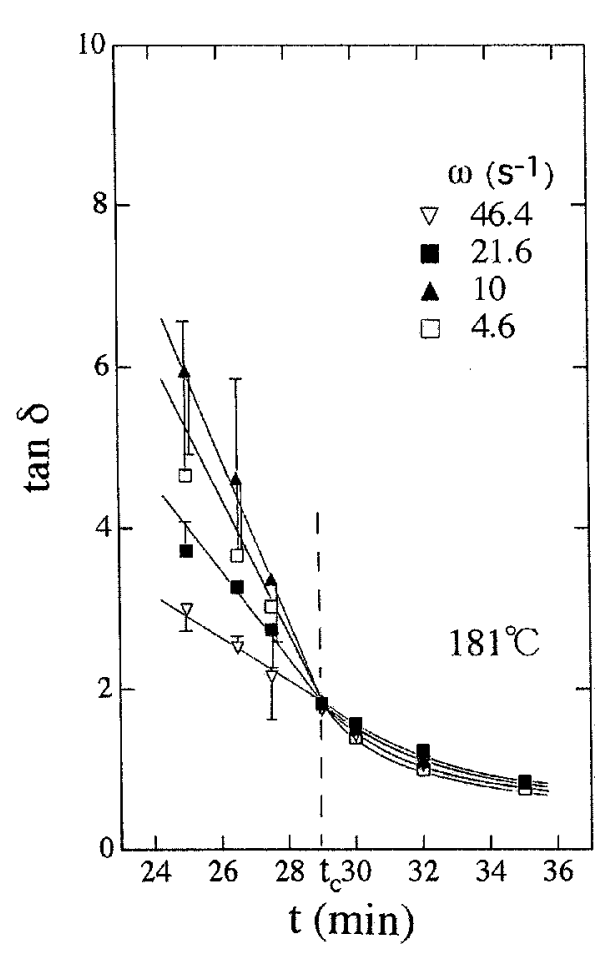

FIG. 3. Change in $\tan \delta$ at fixed $\omega$ 's during the gelation process of the PDTS/PS system at $181{ }^{\circ} \mathrm{C}$, where the vertical dashed line indicates the gelation point $\left(t_{c}=29.0 \mathrm{~min}\right)$ at $181^{\circ} \mathrm{C}$.

the time change in $\tan \delta$, the gelation process can be divided into the following three stages with respect to a characteristic time $t_{c}$ for formation of the critical gel:

(i) At $t<t_{c}, G^{\prime}$ increases more rapidly than $G^{\prime \prime}$, and thus $\tan \delta$ decreases rapidly with $t$. The $\tan \delta$ value is larger at lower $\omega$ 's. In this region, PDTS forms the networks which are not macroscopically percolated yet. The clusters of the networks have the upper cut-off size $\xi$.

(ii) At $t=t_{c}, \tan \delta$ becomes independent of $\omega$. This indicates that the system has reached the gel point and that the clusters of the networks with macroscopically percolated or with infinitely large molecular weight is formed.

(iii) At $t>t_{c}$, the clusters are linked into threedimensionally interconnected networks. $G^{\prime}$ still increases faster than $G^{\prime \prime}$, but its growth rate becomes moderate. As a result, $\tan \delta$ decreases gradually with $t$.

We can calculate the values of $n$ from $\tan \delta$ at $t_{c}$ by using Eq. (17). The values of $t_{c}, \tan \delta$ at gel point, and the exponent $n, n_{\tan }$, obtained from the $\tan \delta$ at gel point at each gelation temperature are summarized in Table I.

TABLE I. Characteristic time for formation of the critical gel or gel point $\left(t_{c}\right)$, loss tangent at $t_{c}(\tan \delta)$, the exponent $n\left(n_{\tan }\right.$, obtained from $\tan \delta$ at gel point, or $n_{\text {slope }}$, obtained from $\log _{10} G^{\prime}$ and $\log _{10} G^{\prime \prime}$ vs $\log _{10} \omega$ plot), gel strength $S$, fractal dimension $d_{f}\left[d_{f, \text { ex }}\right.$, obtained from Eq. (15), and $d_{f, \mathrm{sc}}$, obtained from Eq. (16)] at each temperature.

\begin{tabular}{cccccccc}
\hline \hline $\begin{array}{c}\text { Temp. } \\
\left({ }^{\circ} \mathrm{C}\right)\end{array}$ & $\begin{array}{c}t_{c} \\
(\mathrm{~min})\end{array}$ & $\tan \delta$ & $n_{\tan }$ & $n_{\text {slope }}$ & $\begin{array}{c}10^{-2} S \\
\left(\mathrm{Pas}^{n}\right)\end{array}$ & $d_{f, \text { ex }}$ & $d_{f, \text { sc }}$ \\
\hline 183 & 46.8 & 1.84 & 0.68 & 0.68 & 9.93 & 2.41 & 0.95 \\
181 & 29.0 & 1.84 & 0.68 & 0.68 & 19.8 & 2.41 & 0.95 \\
178 & 16.7 & 1.63 & 0.65 & 0.65 & 23.0 & 2.62 & 0.98 \\
\hline \hline
\end{tabular}

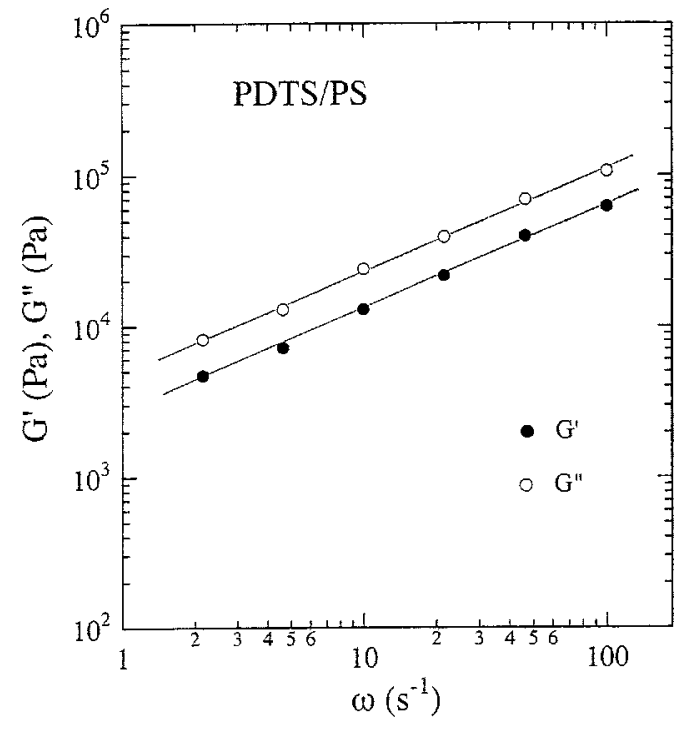

FIG. 4. Shear moduli $G_{c}^{\prime}$ and $G_{c}^{\prime \prime}$ at gel point plotted as a function of $\omega$ in double logarithmic scale for the gelation of the PDTS/PS system at $181{ }^{\circ} \mathrm{C}$. Solid lines indicate the best-fitting results with the power-law behavior with $n=n_{\text {slope }}$ in Table I [Eq. (12)].

\section{Viscoelasticity at the gel point}

Figure 4 shows the frequency dependencies of $G^{\prime}$ and $G^{\prime \prime}$ at the gel point (defined, respectively, by $G_{c}^{\prime}$ and $G_{c}^{\prime \prime}$ ) at $181^{\circ} \mathrm{C}$. In order to obtain $G_{c}^{\prime}(\omega)$ and $G_{c}^{\prime \prime}(\omega)$ at each gelation temperature, we interpolated the values $G^{\prime}(\omega)$ and $G^{\prime \prime}(\omega)$ at $t_{c}$ from the curves of $G^{\prime}(\omega)$ versus $t$ and $G^{\prime \prime}(\omega)$ versus $t$ at each temperature as shown in Figs. 1 and 2, respectively. The plots of $\log G_{c}^{\prime}(\omega)$ and $\log G_{c}^{\prime \prime}(\omega)$ versus $\log \omega$ at each temperature become linear and parallel to each other. Thus $G^{\prime}$ and $G^{\prime \prime}$ obey the power law of $\omega$ as described by Eq. (12). We estimated the values $n, n_{\text {slope }}$, from the plots of $\log G^{\prime}$ and $\log G^{\prime \prime}$ versus $\log \omega$, at each gelation temperature and listed them in Table I. The values of $n_{\text {slope }}$ agree with those of $n_{\text {tan }}$, indicating that the experimental results confirm theoretical consistency. The values of $n$ thus estimated are almost independent of temperature. We calculated the fractal dimension by using Eqs. (15) and (16) with $n$. The fractal dimensions $d_{f \text {,ex }}$ calculated with Eq. (15) and $d_{f, \text { sc }}$ calculated with Eq. (16) are listed in Table I. The value of fractal dimension $d_{f \text {,ex }}$ agrees with $d_{f}=2.5$ calculated under the case where the percolation theory is also valid for physical gelation system such as hydrodynamic interactions are completely screened out and the excluded volume effects are dominant. ${ }^{8}$ However, it is not clear that the case is valid in the PDTS/PS system. Thus we need to determine the fractal dimension directly by using scattering measurement.

In Table I, the gel strength $S$ calculated with Eq. (13) is also listed. The gel strength increases with decreasing temperature. We will discuss the relationship between $S$ and the structure of the gel later.

\section{Scattering profiles at gel point}

Figure 5 shows the scattered intensity of USAXS, and SAXS at gel points for each measurement temperature. We shifted SAXS profiles vertically in a double logarithmic plot 


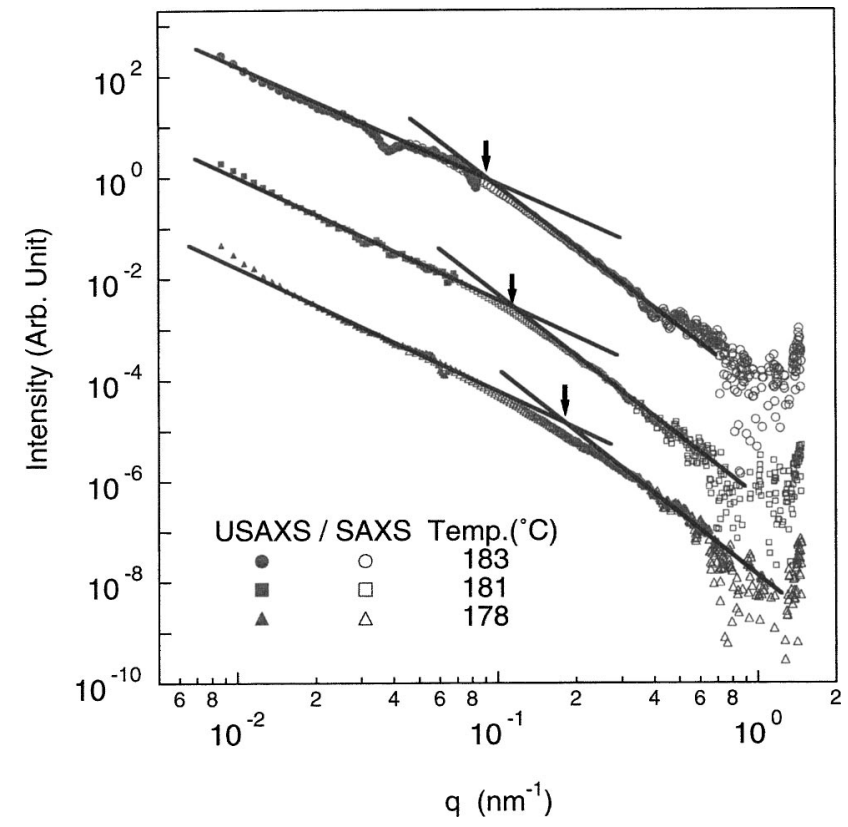

FIG. 5. Scattered intensity profiles of USAXS and SAXS for PDTS/PS system at 178,181 , and $183^{\circ} \mathrm{C}$. USAXS profiles at 181 and $178^{\circ} \mathrm{C}$ are shifted vertically by factors of 100 and 10000 , respectively. SAXS profiles are shifted vertically to be connected to the corresponding USAXS profiles smoothly. Note that the USAXS and SAXS profiles are overlapping over a sufficiently large $q$ range. Arrows indicate the crossover point $q_{c}$ of the scattered intensity or the inverse of the lower limit of fractality in length scale.

to connect SAXS profiles to USAXS profiles smoothly. The scattering profiles of the PDTS/PS at gel point can be divided into the two $q$ regions at crossover wave number $q_{c}$ indicated by the arrows in the figure.

Above $q_{c}$, the scattered intensity obeys Porod law or $q^{-4}$, which reflects a sharp interface of the fibril made of the crystalline structure of the PDTS packed hexagonally ${ }^{14}$ in PS matrix. Below $q_{c}$, the scattered intensity obeys the power law of $q^{-m}$ with $m$ being about 2.5. This power law means that the PDTS crystalline fibrils form the self-similar structure with mass-fractal dimension $d_{f}$ being $m .{ }^{15}$ The values of $d_{f, \text { sf }}$ obtained from scattered intensity at each measurement temperature are listed in Table II. The fractal dimension $d_{f, \text { sf }}$ agrees with $d_{f \text {,ex }}$, as listed in Table I, indicating that hydrodynamic interactions are completely screened out and that the excluded volume effects are dominant in the PDTS/PS gel system. The value of $d_{f, s f}$ slightly increases with decreasing temperature as listed in Table II, indicating that the more dense network with winding path was formed with decreasing temperature. $q_{c}$ corresponds to the inverse of the lower cut-off length scale of the fractality and increases with decreasing temperature. The difference in $q_{c}$ affects the gel

TABLE II. Fractal dimension $d_{f}, d_{f, \text { sf }}$, obtained from the scattered intensity, and crossover wave number $q_{c}$.

\begin{tabular}{ccc}
\hline \hline Temp. $\left({ }^{\circ} \mathrm{C}\right)$ & $d_{f, \mathrm{sf}}$ & $q_{c}\left(\mathrm{~nm}^{-1}\right)$ \\
\hline 183 & 2.40 & 0.11 \\
181 & 2.49 & 0.13 \\
178 & 2.58 & 0.22 \\
\hline \hline
\end{tabular}

strength. Scanlan and Winter ${ }^{16}$ investigated the changes in the gel strength $S$ of end-linked poly(dimethylsoloxane) (PDMS) with stoichiometry, concentration, and molecular weight at gel point and found that $S$ is expressed by

$$
S=G_{0} \lambda_{0}^{n},
$$

where $G_{0}$ and $\lambda_{0}$ are, respectively, the characteristic modulus and the short time constant. As for the gel of end-linked PDMS, $G_{0}$ and $\lambda_{0}$ are, respectively, close to the plateau modulus of prepolymer of PDMS and the longest relaxation time of prepolymer. As for the gel of PDTS/PS system, the decrease in the crossover length scale $l_{c}=q_{c}^{-1}$ corresponds to the decrease in the molecular weight between crosslink points in the case of a chemical gel such as PDMS gel. The decrease in $l_{c}$ is thought to cause the increase in $G_{0} \cdot \lambda_{0}$ changes with temperature due to the change in the friction coefficient. However, those effects may be somewhat small, because $n$ in Eq. (18) is smaller than unity. Anyway, the increase in $S$ with quench depth originates mainly from the decrease in $l_{c}$.

\section{CONCLUSION}

We investigated the physical gelation process of PDTS/PS system by using viscoelastic and scattering experiments. We measured time evolution of $G^{\prime}$ and $G^{\prime \prime}$ in the physical gelation process of PDTS/PS system. At the gel point, $G^{\prime}$ and $G^{\prime \prime}$ were expressed by the power law of $\omega$, $G^{\prime} \sim G^{\prime \prime} \sim \omega^{n}$, with the critical exponent $n$ being nearly equal to $2 / 3$. The gel strength increases with the quench depth. We investigated the structure factor over two decades in length scale at gel point by using ultra-small-angle X-ray scattering, and small-angle X-ray scattering. We found the power-law behavior $q^{-d_{f}}$ in low- $q$ region, indicating that the gel network forms the self-similar structure with mass-fractal dimension $d_{f} . d_{f}$ is nearly equal to 2.5 and agrees with the fractal dimension calculated from the exponent $n$ with the condition that hydrodynamic interactions are completely screened out and the excluded volume effects are dominant in the gel. The gel strength was found to increase with the decrease in the lower limit length scale of fractality.

\section{ACKNOWLEDGMENT}

This work was supported, in part, by a Grant-in-Aid from Japan Society for the Promotion of Science (Grant Nos. 15540392 and 14045245).

${ }^{1}$ T. Kobayashi, H. Hasegawa, and T. Hashimoto, J. Soc. Rheol., Jpn. 17, 86 (1989).

${ }^{2}$ M. Takenaka, T. Kobayashi, T. Hashimoto, and M. Takahashi, Phys. Rev. E 65, 041401 (2002).

${ }^{3}$ T. Hashimoto and N. Sakamoto, Macromolecules 28, 4779 (1995)

${ }^{4}$ T. Kobayashi, H. Yagi, S. Kitagawa, and T. Hashimoto, Bull. Chem. Soc. Jpn. 7, 850 (1993).

${ }^{5}$ T. Kobayashi, M. Takenaka, and T. Hashimoto (unpublished).

${ }^{6}$ T. Fujisawa, K. Inoue, T. Oka, H. Iwamoto, T. Uruga, T. Kumasaka, Y. Inoko, N. Agi, M. Yamamoto, and T. Ueki, J. Appl. Crystallogr. 33, 797 (2000)

${ }^{7}$ T. Koga, M. Hart, and T. Hashimoto, J. Appl. Crystallogr. 29, 318 (1996).

${ }^{8}$ J. E. Martin, D. Adolf, and J. P. Wilcoxon, Phys. Rev. A 39, 1325 (1989). 
${ }^{9}$ M. Rubinstein, R. H. Colby, and J. R. Gillmor, Polym. Prepr. (Am. Chem. Soc. Div. Polym. Chem.) 30, 81 (1989); in Space-'lime Organization in Macromolecular Fluids, edited by F. Tanaka, M. Doi, and T. Ohta (Springer, Berlin, 1989).

${ }^{10}$ M. Muthukumar, I. Chem. Phys. 83, 3162 (1985).

${ }^{11}$ M. Muthukumar, Macromolecules 22, 4656 (1989).

${ }^{12}$ W. Hess, T. A. Vilgis, and H. H. Winter, Macromolecules 21, 2536 (1988).
${ }^{13}$ J. D. Ferry, Viscoelastic Properties of Polymers, 3rd ed. (Wiley, New York, 1980)

${ }^{14}$ T. Kobayashi, M. Takenaka, and T. Hashimoto, KOBUNSHI RONBUNSHU 55, 613 (1998).

${ }^{15}$ D. W. Schaefer and K. W. Keefer, Mater. Res. Soc. Symp. Proc. 32, 1 (1984); J. E. Martin and A. J. Nurd, J. Appl. Crystallogr. 20, 61 (1987); P. W. Rouw and C. G. de Kurif, Phys. Rev. A 39, 5399 (1989).

${ }^{16}$ J. C. Scanlan and H. H. Winter, Macromolecules 24, 47 (1991). 